\title{
Research On Springback Control Based On GRNN-ES
}

\author{
Zhaohu Deng ${ }^{1,2, a}$ \\ ${ }^{1}$ School of Mechanical and Automotive Engineering South China University of Technology \\ Guangzhou, China \\ ${ }^{2}$ Mechanical and Electrical Engineering Department Guangdong Polytechnic College Guangzhou, \\ China \\ a360596374@qq.com
}

Keywords: springback control; ANN; ES; optimization

Abstract. Springback is a kind of formation defect in punch machining. It can be decreased by adjusting the processing parameters. In this paper it put forward to optimize the parameters with response surface method. Firstly it built an artificial neural network for mapping the relation between the parameters and springback. Then it optimized the neural network model with evolution strategy. At last it would get the optimum of parameters after optimization. In order to get more precise parameters it modified the ANN and ES in this paper. Then it took a trial to certify the method. The results showed that the method proposed in this paper can decrease the springback effectively.

\section{Introduction}

Sheet metal forming is a kind of important machining method. So it is applied in automobile, appliance and aerospace manufacture widely. But when the sheet is in forming, the phenomenon-springback will influence the products' precision seriously[1]. The formation error will lead to that the product can't be assembled. With the development of automobile industry and aerospace industry, the requirement on formation precision goes high more and more. Especially when the high strength steel sheet and aluminum alloy sheet are much in use, the problem of springback becomes more difficult to solve. So how to control the springback effectively has been the critical technology which influences the precision punch and high-precision mould design.

Research showed that processing parameters such as material properties, sheet thickness, friction ect influence the springback very much. It can decrease the springback by adjusting the parameters. So in order to minimize the springback choosing the optimum parameters has been a practical method[2].

\section{Research on springback control}

Method of springback control. When the sheet is under load, it would deform in the direction of its application. But after the load is taken off, the sheet would deform in the opposite direction because of the residual stress. This phenomenon is called springback. So it can be seen that the springback relates to material property. When the elastic module is higher, the ability of sheet resisting elastic deformation becomes much stronger, the springback will be lower. When the yield strength is higher, the required load is higher; the springback will be high too. At the same time the blank thickness influence the springback as well. When the sheet is thicker, the ratio of elastic deformation is lower, the springback is lower.

And the springback still relates to processing parameters. For example when the friction force is high, it will increase the stress zone of sheet. Then the stress state of sheet's two surfaces will be in consistent. The springback will be decreased. When the punching force increases, the ratio of plastic deformation will increase too. Then the springback will be decreased. According to the analysis above it is known that the springback can be decreased by setting the parameters properly. However the relation between the parameters and the springback is so complicated that it can not be expressed how the parameters influencing the springback with one exact function now. So how to choose the optimum parameters is still in further developing. At present the most common methods include trial and error, 
orthogonal trial analysis, response surface method ect. In this paper it adopted one kind of response surface method which was based on ANN (artificial neutral network) and EA (evolutionary algorithm). In this method it firstly built the mapping relation between the processing parameters and springback with ANN. And then it would optimize the trained ANN by ES (Evolutionary Strategies). At last it would get the optimum processing parameters which could make the sprinback of sheet minimal.

Mapping model of springback. Because the mapping relation between the processing parameters and springback is very complicated, it can't be expressed with explicit function. In this paper it supposed to build a RBF neural network to mapping the relation. RBF neural network has the ability to mapping the relation between the input and output. And researches showed that RBF neural network was one of the best mapping neural networks in feed-forward ANN[3]. So the RBF neural network has been applied in engineering field widely. But when building the RBF neural network, the distribution of neural cells depend on original samples too much. The cells' distribution will trap into the local optimum. So in this paper it introduces the niche genetic algorithm for its characteristic of global optimization. The whole process of building model was divided into two sections. In section one it was supposed to build a rough RBF neural network with GA. It took the cells as optimized variable and the performance function as target function. Thus it can avoid the local optimization of cells' distribution. Because the GA has the deficit of premature, it proposed to bring niche factor to keep the population variety at early stage. And at the later stage of optimization it would define the niche factor as zero to improve the convergence speed of GA. Because GA's ability of optimizing in local area is worse than in global, in section two it mainly refined the RBF neural network built in section one with gradient method.:

Optimization of processing parameters. As to the ANN the traditional optimization method such as simplex search method or lagrangian multiplier method doesn't work. It only can be optimized by EA or other modern optimization methods. In this paper the new built neural network will be optimized with ES (evolution strategy). ES was kind of evolution algorithm as well as GA. It allows the variables in decimal style. So it can get more precise results for continuous function.

For carrying out the mutating operation conveniently, the individuals of ES were defined as binary expression (i.e the individuals consist of target variable $\mathrm{X}$ and standard deviation $\sigma$ ) traditionally. In this paper it introduced a third variable $\alpha$ - rotating angle of coordinate. Fig. 1 showed the effect of three variables. (a) is the situation when $\sigma$ is equal, $\alpha$ is zero. The variation range of individual is in circle area. (b) is the situation when $\sigma$ is unequal, $\alpha$ is zero. The variation range of individual is in ellipse area. (c) is the situation when $\sigma$ is unequal, $\alpha$ works. The variation range of individual is also in ellipse area. But here the ellipse can rotate. It meant that the variation of individual were more free. The individual had more probability to reach the optimum.

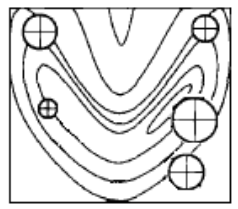

(a)

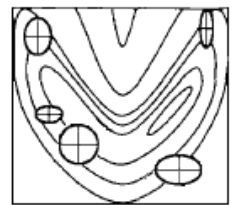

(b)

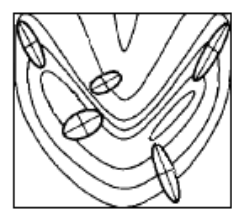

(c)

Fig. 1 The effect of $\sigma$ and $\alpha$

The steps of building ES:

1)Initiate the parameters of ES.And then define the expression of target function and the individual

2) Define the original cluster with $\mu$ individuals and calculate the fitness of every individual

3) Judge if the original fitness meet the accuracy or not: yes, to output the optimum. no, to go to the next step

4) Arraying the every component of individual for producing the new individual. Then mutate the individuals with mutation operator for $\lambda$ times to produce $\lambda$ individuals.

5) Calculate the fitness of the $\lambda$ individuals. And take a comparison to choose the best $\mu$ one. 
6) Repeat the step 4 and 5 until the iteration reach set value or requirement of accuracy is met. Then quit the program and output the optimum individuals.

In order to certify the performance of ES, it choose one test function as Fig.2 shown. At the same time it compared three-component ES with two-component ES wiht that function

$f=0.5+\left(\left(\sin \left(\operatorname{sqrt}\left(x^{2}+y^{2}\right)\right)\right)^{2}-0.5 x^{2}+y^{2}\right) /\left(1+0.001\left(x^{2}+y^{2}\right)\right)^{2}$

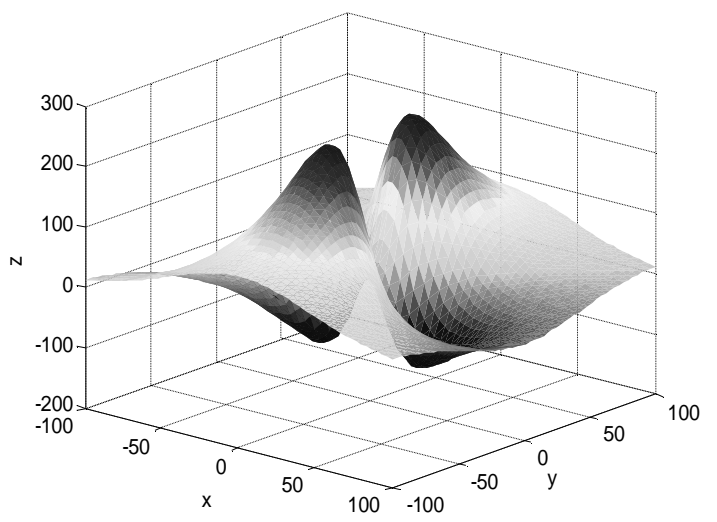

Fig. 2 The test function

It took 333 times to get the optimum with two-component ES. The optimum is -124.4920 . Fig. 3 is the route of individuals searching for optimum. Fig 4 shows the fitness's variation of every iteration. From the figure it can be seen that the algorithm can get the optimum, but the convergence speed was slow.

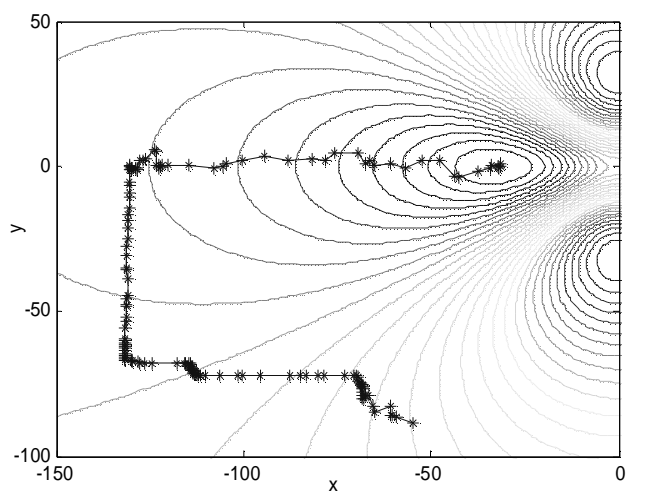

Fig.3 The path of optimum seeking

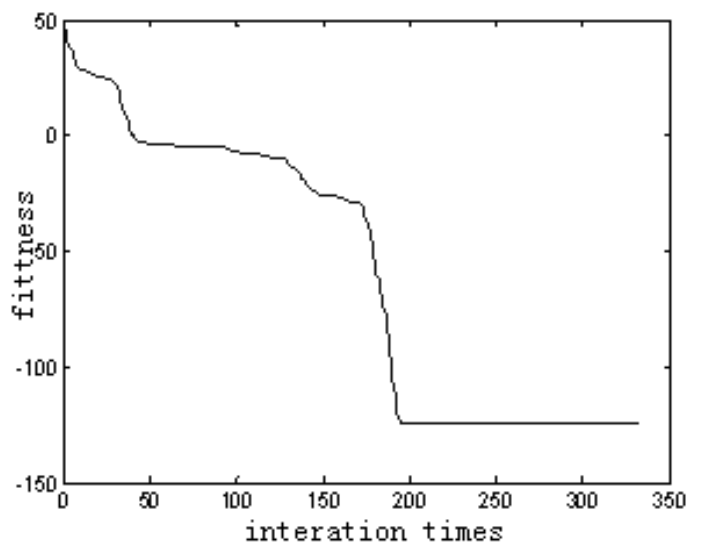

Fig.4 The curve of sufficiency function changing

It took 137 times to get the optimum with three-component ES. The optimum is -124.4964. Fig.5 is the route of individuals searching for optimum. Fig.6 shows the fitness's variation in every iteration. Compared to the two-component ES the modified one had better convergence.

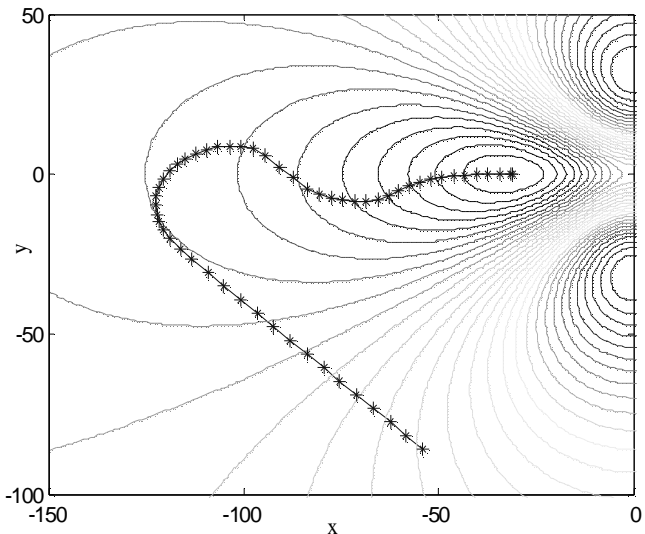

Fig. 5 The path of optimum seeking

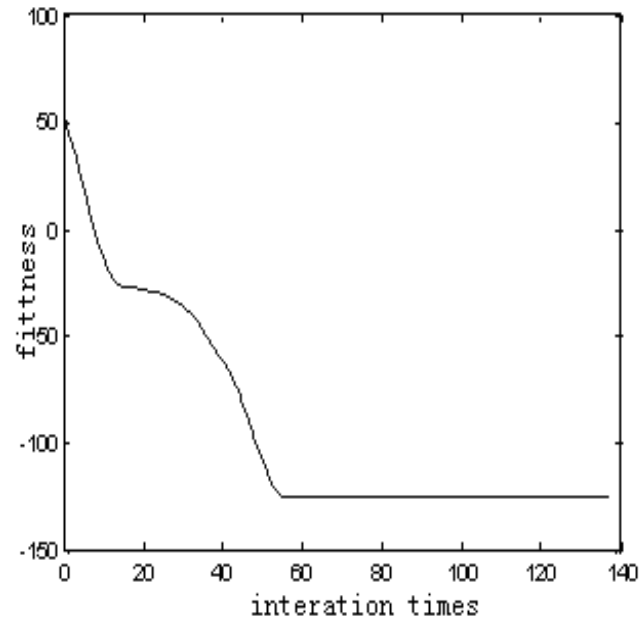

Fig.6 The curve of sufficiency function changing 


\section{The application of response surface method}

Sheet metal curling can be divided into two steps. At second step it involved second formation and reverse load, so the springback was complicated. It can't build the relation between the parameters and the springback with explicit function. That made it difficult to control the springback with optimization. In this paper it proposed to solve the problem with response surface method. It firstly built the relation between the parameters and the springback with GRNN. Secondly it optimized the trained GRNN with three-component ES. After the optimization it would get the optimum parameters.

According to the analysis above there were many parameters influencing the springback. In this paper it took material properties, thickness, punching force and friction state as the input variable of GRNN. Fig. 10 was the certificated results. The most relative error was $6.42 \%$. the least relative error was $0.93 \%$. It showed that the prediction of GRNN consist with the true valve very much.

Define the trained GRNN as target function, the parameters as optimized variable. The size of original cluster $\mu=5$, the size of cluster after mutation $\lambda=35$.

After optimization it got the following parameters as optimum:1.5mm DC54D steel sheet, non-lubricated state, punching force is equal to $15 \mathrm{KN}$. Then it carried out a trial with the optimum parameters. The results showed that the springback is $12.16 \times 10^{-4} \mathrm{~mm}^{-1}$. Compared to other optimization method, it showed that it can decrease the springback effectively with response surface method.

\section{Conclusions}

If you follow the "checklist" your paper will conform to the requirements of the publisher and facilitate a problem-free publication process.It proposed a method of optimizing the processing parameters, which involved GRNN prediction model and modified ES. Trials showed that this method can effectively search the optimum and decrease the springback.According to the problem the cells' distribution of RBFNN trapped into local optimization, it distributed the cells with GA. At the same time it adopted the niche factor for improving the ability of total searching. Certification showed that the built GRNN can mapping the non-linear relation exactly.

For improving the precision of optimization it proposed to take ES as the optimization method. For improving the speed of optimization, it introduced a third variable-coordinate rotating angle. The certification showed that the modified ES can search the optimum quickly.

\section{Acknowledgements}

This work was financially supported by the Project of Research and Production Combination Financed by Guangzhou. Project number: 201392-00084.

\section{References}

[1] Jingxin Chen, Lan Cai and Guomin Lu, etl:Automotive Engineering Vol.27(2005),p.118.

[2] Yanfang Liu, Fazhong, Shi:Forging \& Stamping Technology Vol.4(2004),p.36.

[3] Qingsheng Xie: The method of neural networks applied in mechanical engineering (Mechanical Industry Publications, China 2003). 